\title{
Risk factors for severe COVID-19 disease and death in patients aged 70 and over: a retrospective observational cohort study
}

Katleen Fagard, Evelien Gielen , Mieke Deschodt , Els Devriendt \& Johan Flamaing

To cite this article: Katleen Fagard , Evelien Gielen , Mieke Deschodt , Els Devriendt \& Johan Flamaing (2021): Risk factors for severe COVID-19 disease and death in patients aged 70 and over: a retrospective observational cohort study, Acta Clinica Belgica, DOI: 10.1080/17843286.2021.1890452

To link to this article: https://doi.org/10.1080/17843286.2021.1890452

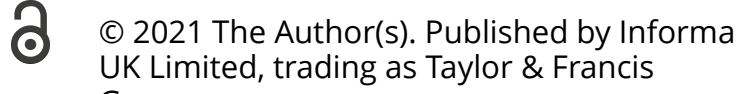
Group.

\section{Published online: 21 Feb 2021.}

Submit your article to this journal $₫$

Џlll Article views: 188

Q View related articles $₫$

View Crossmark data $\circlearrowright$ 


\title{
Risk factors for severe COVID-19 disease and death in patients aged 70 and over: a retrospective observational cohort study
}

\author{
Katleen Fagard $\mathbb{D}^{\mathrm{a}, \mathrm{b}}$, Evelien Gielen $\mathbb{D}^{\mathrm{a}, \mathrm{b}}$, Mieke Deschodt $\mathbb{D}^{\mathrm{b}, \mathrm{c}}$, Els Devriendt ${ }^{\mathrm{a}}$ and Johan Flamaing $\left(\mathbb{D}^{\mathrm{a}, \mathrm{b}}\right.$

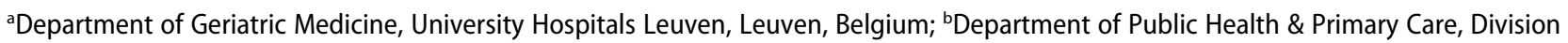 \\ of Gerontology and Geriatrics, KU Leuven, Leuven, Belgium; CHealthcare and Ethics, Faculty of Medicine and Life Sciences, UHasselt, \\ Hasselt, Belgium
}

\begin{abstract}
Objectives: The COVID-19 pandemic resulted in rapid reorganisations of hospital care. In our hospital, the geriatrics team introduced the Clinical Frailty Scale (CFS) on the non-ICU COVID-19 units during these reorganisations. A retrospective analysis was performed to investigate the CFS as a risk factor for severe COVID-19 disease and in-hospital death in older patients with COVID-19.

Methods: In patients aged $\geq 70$ years, an online geriatric assessment questionnaire was launched, from which the CFS was scored by the geriatrics team. Additional clinical data were collected from the electronic medical records. Risk factors related to ageing, such as the CFS, age-adjusted Charlson Comorbidity Index, living situation and cognitive decline, were examined alongside frequently reported risk factors in the general population. Outcomes were in-hospital death (primary outcome) and oxygen need of $\geq 6$ litres and early warning score $\geq 7$, as parameters for severe disease (secondary outcomes). Baseline characteristics were described with descriptive statistics. Associations were analysed with uni- and multivariable analyses.

Results: One hundred and five patients were included, median age 82 years. CFS scores were 1-4 in 43, 5-6 in 45, and 7-9 in 17 patients. In multivariable analysis, CFS and cognitive decline were the only risk factors that were independently associated with in-hospital mortality. Chronic obstructive pulmonary disease, presence of respiratory symptoms on admission and male gender showed and independent association with severe disease.

Conclusion: A retrospective analysis shows that CFS and cognitive decline have added value for predicting in-hospital mortality in older patients with COVID-19 disease.
\end{abstract}

KEYWORDS

Aged; cohort studies; COVID19; frailty; prognostic factors

\section{Introduction}

In December 2019 several pneumonia cases of unknown origin emerged in China, Wuhan. On January 9, 2020 a novel virus was detected as the causative agent [1]. The virus was highly similar to the Coronavirus (CoV) that caused an outbreak of severe acute respiratory syndrome (SARS) in 2003. Thus, it was named SARS-CoV-2 by the World Health Organization (WHO), and the associated disease was named COVID-19 disease [2]. By the end of January 2020 the first European cases were reported and by March 11 COVID-19 was declared a global pandemic by the WHO [1].

It soon became clear that the pandemic would have an enormous impact on health care resources. As it was feared that there would be insufficient hospital capacity to deal with all COVID-19 patients during the ascending curve, guidelines with triage criteria for hospitalisation or admission to an intensive care unit (ICU) were issued to prioritise those most likely to survive [3-5]. The guidelines included flow-charts with characteristics such as disease presentation, age, comorbidities and frailty, and based identification of frailty on the Clinical Frailty Scale (CFS). The CFS was developed and validated by Rockwood et al. in 2005 and showed an increasing risk of death and institutionalisation with increasing frailty [6]. The rationale for including the frailty concept is clear: avoid undertreatment based on age and also disproportionate care for frail older patients. Published studies in ICU patients have shown that CFS is better associated with mortality in severe disease than age [7].

The clinical spectrum of COVID-19 disease ranges from asymptomatic to severe respiratory failure, multi-organ failure and death $[8,9]$. For well-founded clinical decisions, it is important to identify prognostic factors related to more severe disease and mortality. Several observational cohort studies examining risk factors for severe COVID-19 disease and death in the general population have been published. Apart from older age, the main risk factors described are male gender, obesity, and comorbidities such as hypertension, diabetes, cardiovascular disease, chronic pulmonary disease, cancer, chronic kidney disease [10-12]. Hereafter we call them 'conventional' risk

CONTACT Katleen Fagard katleen.fagard@uzleuven.be Department of Geriatric Medicine, University Hospitals Leuven, Gasthuisberg, Herestraat 49, Leuven B-3000, Belgium 
factors. Publications studying risk factors such as frailty and comorbidity indices in COVID-19 patients were non-existing when we initiated the frailty screening on the non-ICU COVID-19 units. Because of this knowledge-gap we decided to study the CFS, the Charlson Comorbidity Index (CCl), age-adjusted $\mathrm{CCl}$, living situation and cognitive decline as 'ageingrelated' risk factors for severe COVID-19 disease and death in addition to the 'conventional' risk factors named above.

In this study, we investigate the association between 'ageing-related' and 'conventional' risk factors with in-hospital mortality, high oxygen need ( $\geq 6$ litres), and high early warning scores (EWS $\geq 7$ ) in patients aged 70 and over admitted to a COVID-19 hospitalisation unit.

\section{Methods}

\section{Study design, setting and sample}

A single-centre retrospective observational cohort study was conducted in the University Hospitals (UH) Leuven in Belgium. Patients hospitalised with COVID-19 disease that had a Clinical Frailty Score performed between March 16 and May 16, 2020 were eligible for inclusion.

The diagnosis of COVID-19 disease was made based on a positive polymerase chain reaction (PCR) test or, in the absence of a positive PCR test, based on the clinical picture and a chest CT scan. Patients that screened negative on the PCR test with an alternative diagnosis, e.g. heart failure, bacterial pneumonia, after admission to the COVID-19 hospitalisation unit were excluded from the analysis. The medical ethics committee of the UH Leuven approved the study (S64222). The study is reported according to the STROBE guidelines.

\section{Data collection procedures}

Co-workers of the low-care COVID-19 hospitalisation units (non ICU-units) were instructed to contact the families of newly admitted patients aged $\geq 70$ years by telephone. The patients were either admitted to the lowcare unit immediately from the emergency department, from another hospital unit or after a stay in the ICU. Their family was asked to complete an online geriatric assessment questionnaire and to report the situation before admission and illness. From the questionnaire, the CFS was scored by the geriatrics team. The CFS and the geriatric assessment were reported in the electronic medical record. Data for this study were collected retrospectively from the electronic medical records.

\section{Variables and measurements}

The following demographic data were collected: age, gender, living situation.
The following clinical data were collected: Body Mass Index (BMI) [13], symptoms on admission (flulike symptoms or fever, respiratory symptoms, gastrointestinal symptoms), treatment for COVID-19, a history of cardiovascular disease, chronic pulmonary disease, cancer, hypertension, the $\mathrm{CCl}$ (a comorbidity index that is calculated based on weighted scores assigned to the following conditions: myocardial infarction, chronic heart failure, peripheral vascular disease, cerebrovascular accident or transient ischemic attack, chronic cognitive deficit, chronic obstructive pulmonary disease (COPD), connective tissue disease, peptic ulcer disease, liver disease, diabetes, hemiplegia, chronic kidney disease, solid tumour, leukaemia, lymphoma, acquired immunodeficiency syndrome) [14], and the CFS [6]. In addition to the CCl the ageadjusted $\mathrm{CCl}$ was calculated [15]. A history of cognitive decline reported on the electronic geriatric assessment questionnaire was regarded as presence of cognitive decline and was classified as 'chronic cognitive deficit' in the $\mathrm{CCl}$. Apart from a history of diabetes the glycated haemoglobin value was recorded.

The following outcome variables were collected: Inhospital death (primary outcome), and severe disease, defined in two ways: an oxygen need of 6 litres or more and an EWS of 7 or more during hospitalisation (secondary outcomes) [16].

\section{Data analysis}

Continuous variables were reported as medians with interquartile ranges (IQR). Categorical variables were reported as numbers and percentages. Univariable analyses were performed, comparing patient outcome groups on demographic and clinical variables. Dichotomous and nominal variables were analysed using Pearson Chi-squared tests, or Fisher's exact tests if $\geq 1$ cell had an expected count of less than 5 . Ordinal and continuous variables were analysed using MannWhitney $U$ tests. Multivariable logistic regression models were used to determine independent predictors for the primary and secondary outcomes. Variables showing significant association with the outcome variable in univariable analysis were entered in a multivariable forward stepwise selection procedure. P-values, odds ratios (OR), and $95 \%$ confidence intervals $(\mathrm{Cl})$ are reported. All tests were 2-tailed, assuming a 5\% significance level. All analyses were performed using SPSS version 20.0 (Statistical Package for the Social Sciences Inc., Chicago, Illinois).

\section{Results}

\section{Description of the sample}

Only patients that had a CFS performed on admission to the low-care COVID hospitalisation units 


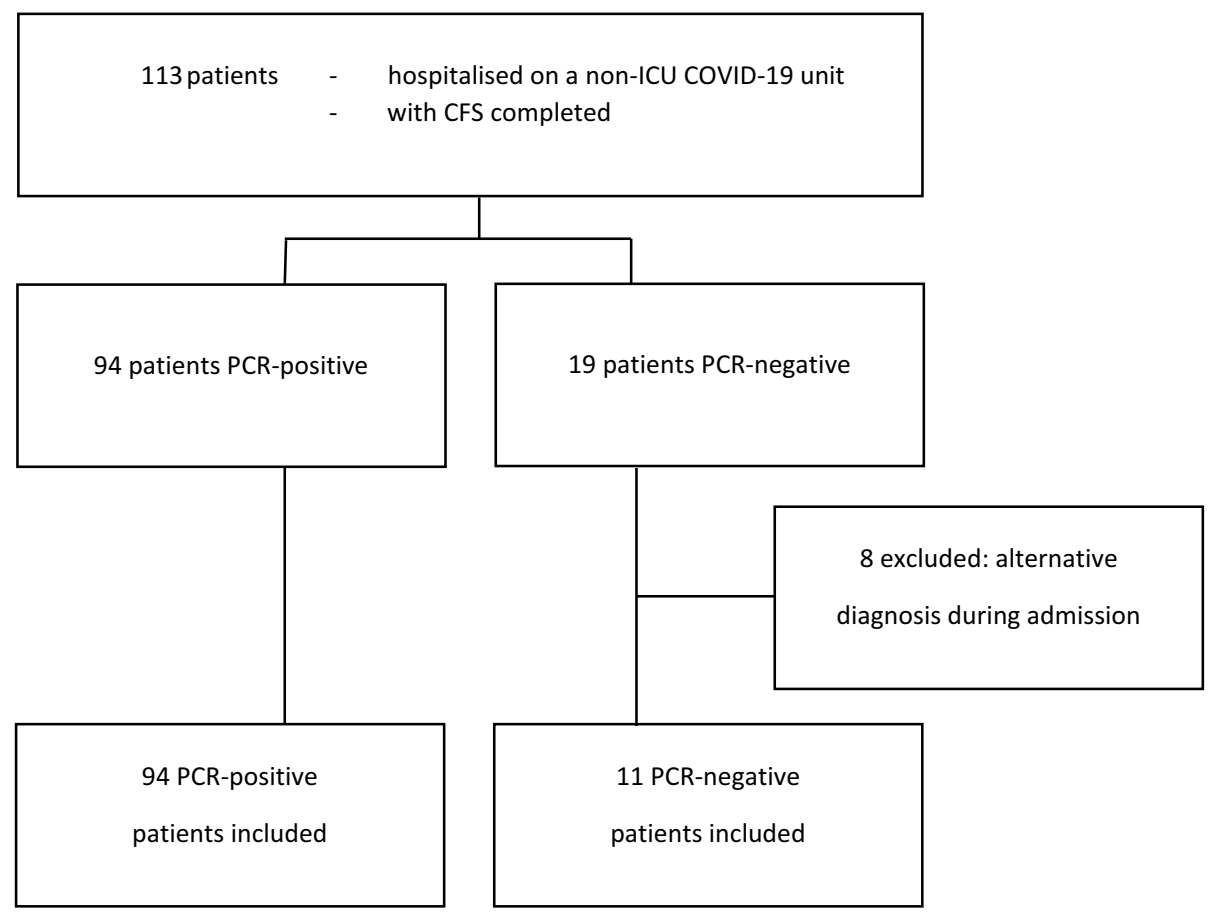

Figure 1. Flow-chart of in- and exclusions. Legend: CFS: Clinical Frailty Scale; PCR: Polymerase Chain Reaction; ICU: Intensive Care Unit;

( $n=113$ ) were eligible for inclusion. Figure 1 displays the in- and exclusion process. One hundred and five patients were included in the study. Patient and clinical characteristics are summarized in Table 1. The median age was 82 years (range 70-97). The male/female ratio was 1.1 . Sixty-two patients lived at home, 10 in an assisted living facility, 33 in a nursing home. CFS scores were 1 or 2 in 13 patients $(12.4 \%), 3$ or 4 in 30 patients $(28.6 \%), 5$ or 6 in 45 patients (42.9\%), and 7 or 8 in 17 patients $(16.2 \%)$. None of the patients had a CFS of 9. Sixtyeight patients had flu-like symptoms or fever, 73 patients had respiratory symptoms and 17 patients complained of gastro-intestinal symptoms on admission. The most common treatment at the time of the study was a combination of hydroxychloroquine (77.1\%) and antibiotics (83.8\%). Eighteen-patients stayed in ICU during admission, of whom 12 were between 70 and 79 years old and 6 between 80 and 85 . The median CFS for patients with ICU transfer was 3.5 (IQR 2-4.25, range 1-6), compared to 6.0 (IQR 4-6, range 1-8) for patients without ICU transfer. Fourteen (13.3\%) patients died during hospitalisation, 40 patients (38.1\%) needed 6 or more litres of oxygen during admission, and 72 patients $(68.6 \%)$ had an EWS score $\geq 7$ during hospitalisation. None of the study patients that were transferred from the low-care COVID unit (i.e. patients in which the CFS was performed) to the ICU died in the ICU. The total ICU mortality for patients aged $\geq 70$ years during the study period, regardless of study participation, was $12 \%(4 / 33)$.

\section{Risk factors for severe disease and death}

Univariable analysis (Table 1) showed that age, CFS, $\mathrm{CCl}$, age-adjusted $\mathrm{CCl}$ and cognitive decline are associated with in-hospital mortality. Living situation, male gender, obesity, cardiovascular disease, chronic pulmonary disease, diabetes, severe kidney disease, cancer and hypertension were not significantly associated. Male gender, presence of respiratory symptoms on admission, and COPD were associated with high EWS $(\geq 7)$; the first two were associated with high oxygen need ( $\geq 6$ litres) during admission. In multivariable logistic regression analysis a higher CFS score and the presence of cognitive decline showed an independent association with in-hospital mortality (Table 2). Multivariable analysis for the secondary outcomes is shown in Table 3 (oxygen need $\geq 6$ litres) and Table 4 (EWS $\geq 7$ ).

Figure 2 shows the relation between the CFS, cognitive decline, and mortality. Mortality rates were higher in patients with CFS 5-6 (7/45, 15.6\%) and CFS $7-9(7 / 17,41.2 \%)$ with no mortality in CFS $1-4(0 / 43$, $0 \%)$. In the deceased group cognitive decline was very prevalent: $92.9 \%$ (13/14) of deceased patients versus $29.7 \%$ (27/91) of patients that survived had reported cognitive decline before admission.

\section{Discussion}

The aim of the current study was to investigate 'ageingrelated' risk factors in older patients, in addition to 'conventional' risk factors for severe COVID-19 disease, regarding their association with severe disease or 


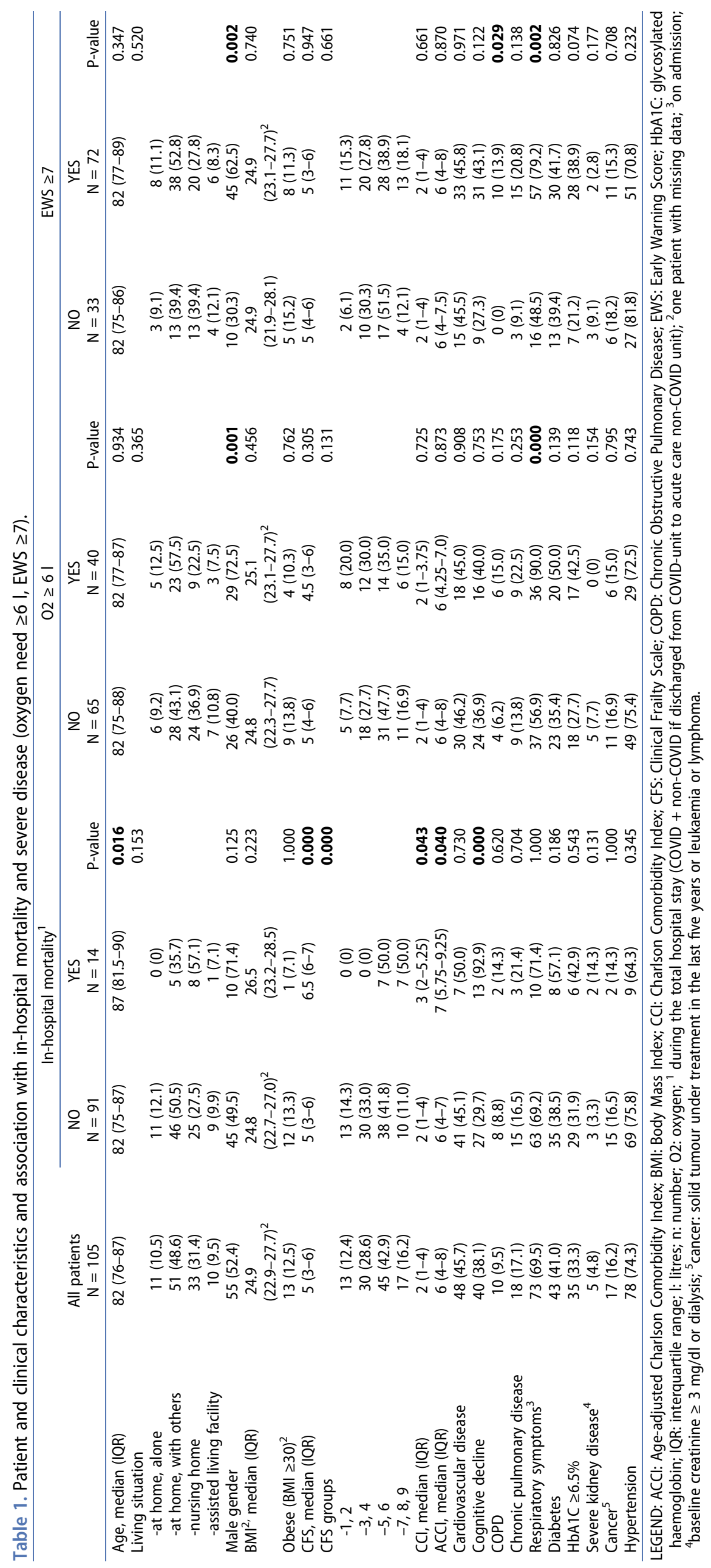


Table 2. Risk factors for mortality: multivariable logistic regression analysis.

\begin{tabular}{lccc}
\hline & \multicolumn{2}{c}{ Univariable analysis } & Multivariable model \\
\cline { 2 - 4 } & OR $(95 \% \mathrm{Cl})$ & P-value & OR $(95 \% \mathrm{Cl})$ \\
\hline Age & $1.113(1.017-1.219)$ & 0.020 & - \\
CFS & $3.243(1.597-6.585)$ & 0.001 & $2.325(1.094-4.941)$ \\
CCl & $1.275(0.990-1.642)$ & 0.060 & - \\
ACCl & $1.277(1.005-1.624)$ & 0.046 & - \\
Cognitive decline & $30.815(3.838-247.419)$ & 0.001 & $11.497(1.317-100.345)$ \\
\hline
\end{tabular}

Legend: ACCI: Age-adjusted Charlson Comorbidity Index; CCI: Charlson Comorbidity Index; CFS: Clinical Frailty Scale; Cl: Confidence Interval; OR: Odds Ratio.

Table 3. Risk factors for 02 need $\geq 6$ litres: multivariable logistic regression analysis.

\begin{tabular}{llcc}
\hline & \multicolumn{2}{c}{ Univariable analysis } & \multicolumn{2}{c}{ Multivariable model } & OR $(95 \% \mathrm{Cl})$ \\
\cline { 2 - 4 } & \multicolumn{1}{c}{ OR $(95 \% \mathrm{Cl})$} & P-value & $3.221(1.315-7.889)$ \\
Male gender & $3.955(1.685-9.282)$ & 0.002 & 0.010 \\
Respiratory symptoms & $6.811(2.170-21.375)$ & 0.001 & $5.622(1.741-18.149)$ \\
\hline
\end{tabular}

Legend: Cl: Confidence Interval; 02: oxygen; OR: Odds Ratio.

Table 4. Risk factors for EWS $\geq 7$ : multivariable logistic regression analysis.

\begin{tabular}{lccc}
\hline & \multicolumn{2}{c}{ Univariable analysis } & \multicolumn{2}{c}{ Multivariable model } \\
\cline { 2 - 4 } & OR $(95 \% \mathrm{Cl})$ & P-value & OR $(95 \% \mathrm{Cl})$ \\
\hline Male gender & $3.833(1.586-9.265)$ & 0.003 & $2.840(1.115-7.234)$ \\
COPD & $\mathrm{N} / \mathrm{A}$ & $\mathrm{N} / \mathrm{A} / \mathrm{A}$-value & 0.029 \\
Respiratory symptoms & $4.037(1.660-9.818)$ & 0.002 & $\mathrm{~N} / \mathrm{A}$ \\
\hline
\end{tabular}

Legend: COPD: Chronic Obstructive Pulmonary Disease; Cl: Confidence Interval; EWS: Early Warning Score; N/A: not applicable, all patients with COPD had an EWS $\geq 7$ which precludes calculating an OR; OR: Odds Ratio

death. We found age, CFS, CCl, age-adjusted CCI and cognitive decline associated with in-hospital mortality. A higher CFS score and the presence of cognitive decline were independent predictors for in-hospital

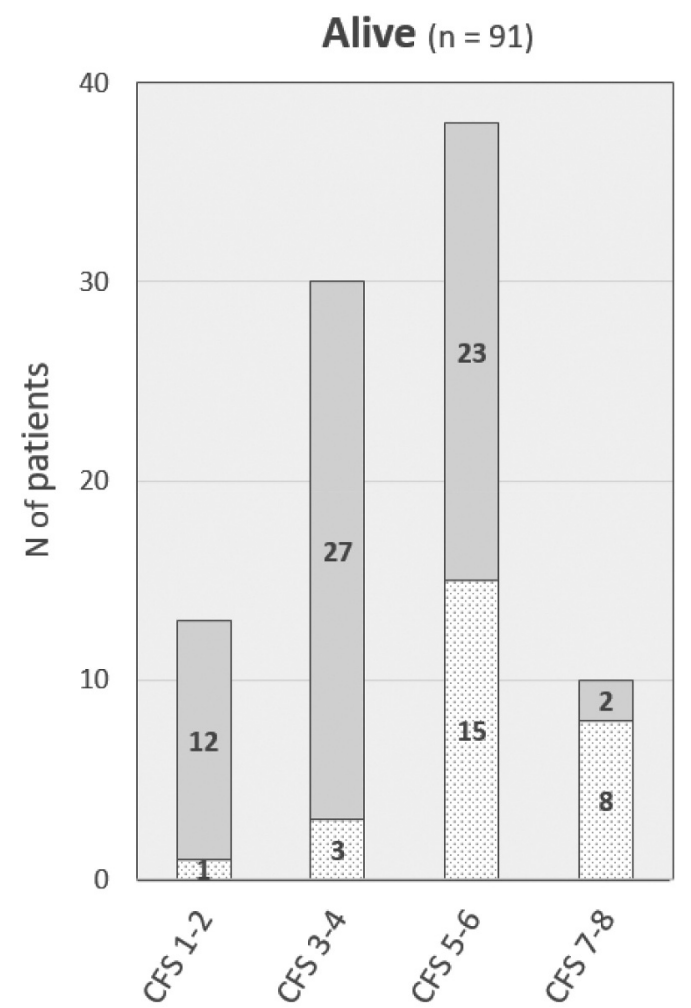

mortality. Living situation, male gender, obesity, cardiovascular disease, chronic pulmonary disease, diabetes, severe kidney disease, cancer and hypertension were not significantly associated. Male gender, presence of

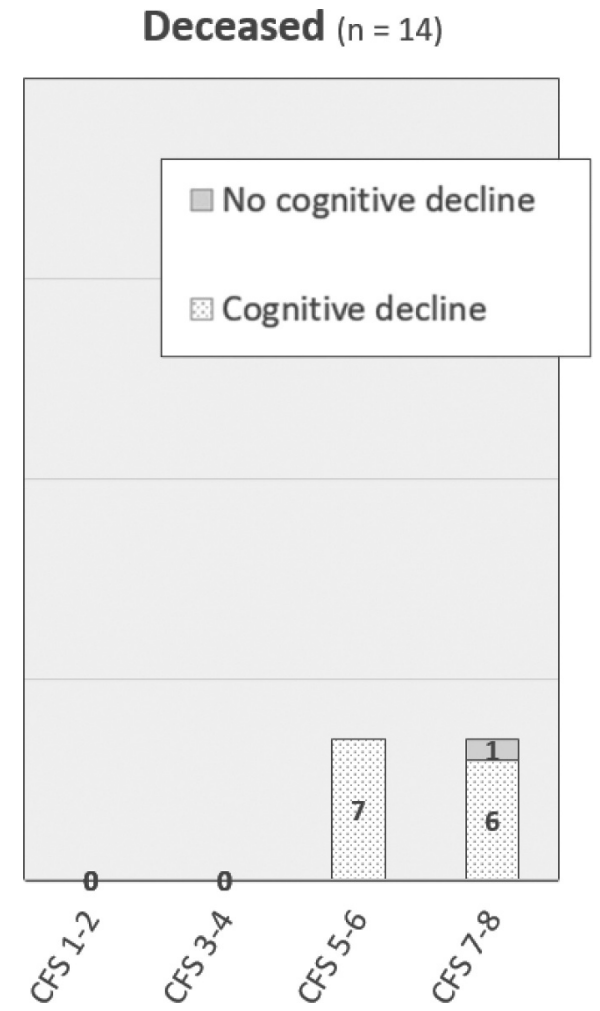

Figure 2. Relation between the CFS score, cognitive decline, and in-hospital mortality. Legend: CFS: Clinical Frailty Scale (note: none of the patients had a CFS score $=9$ ); $\mathrm{N}$ : number. 
respiratory symptoms on admission, and COPD were independently associated with high EWS ( $\geq 7)$; the first two were independently associated with high oxygen need ( $\geq 6$ litres) during hospitalisation.

Previous studies in COVID-19 patients have identified older age and specific comorbidities as risk factors for severe disease and death $[10,12]$. Our study did not find an association with specific comorbidities in older patients, and comorbidity indices and age did not remain as independent predictors for in-hospital mortality in multivariable analysis. Presumably, the impact of ageing and diseases on physical reserves, resulting in frailty, is more important than age and disease alone the older patient population $[17,18]$. In our study, frailty and cognitive decline were more important risk factors for in-hospital mortality than age and comorbidity. Meanwhile, several other studies identified a higher CFS score as a risk factor for mortality [19]. De Smet et al. studied COVID-19 patients on a geriatric hospitalisation unit and found that CFS was independently associated with inhospital ${ }^{1}$ mortality among other risk factors such as age, gender, place of residence, dementia, polypharmacy, radiographic and laboratory findings. With each increase on the frailty scale, the odds ratio for mortality increased with $1.705(95 \% \mathrm{Cl} 1.173-2.750)$. In a bivariate model with age and CFS combined, only the CFS remained significantly associated with mortality. The area under the ROC curve for the CFS in this model was 0.744 ( $95 \% \mathrm{Cl} 0.621-0.867)$, with a positive and negative predictive value of $57 \%$ and $80 \%$ respectively [20]. The largest study, a multicentre European study that included 1564 patients, analysed the effect of frailty on survival in patients with COVID-19 in patients of all ages and concluded that increasing frailty was associated with higher mortality [21]. Compared with CFS 1-2, the adjusted hazard ratios for 7-day mortality were $1.22(95 \% \mathrm{Cl} 0.63-2.38)$ for CFS 3-4, 1.62 (0.81-3.26) for CFS 5-6, and 3.12 (1.56-6.24) for CFS 7-9 [21]. Cognitive decline was most prevalent in the CFS 4-6 group in our study, an indication that patients with cognitive decline and higher frailty scores might not have been transferred to the hospital. It is likely that patients with dementia and severe COVID-19 disease received palliative care in their home environment. In contrast to our study, De Smet et al. did not find a significant association between a diagnosis of dementia and in-hospital mortality ( $p=0.77)$ [20]. This might be due to the difference in operationalisation of dementia. While De Smet et al. only included dementia diagnoses reported in the medical history, we considered all patients of whom the relatives reported cognitive decline in the online assessment questionnaire as patients with cognitive decline. Covino et al. studied patients aged 80 years and over admitted to hospital with COVID-19 disease and concluded that severe dementia was an independent risk factor for 30-day mortality [22]. Cognitive decline is likely to be associated with poorer outcomes because of underlying frailty, less compliance with safety measures and treatments, and risk of delirium. Another explanation is that patients with dementia more often have had advance care planning with higher therapeutic restriction codes and that patients with cognitive decline are less often transferred to ICU.

A systematic review by Jain et al. that reports seven studies, including 1813 COVID-19 patients of all ages, identified dyspnoea $(p<0.001)$, cough $(p=0.04)$, COPD, cardiovascular disease and hypertension (all $p<0.001)$ as predictors for severe disease. They notice that severe COVID-19 disease was not consistently defined across the included studies [23]. It is worth mentioning that the WHO-China joint mission on Coronavirus disease published a report on February 28,2020 in which severe disease was defined as having dyspnoea, respiratory frequency $\geq 30 /$ minute, blood oxygen saturation $\leq 93 \%, \mathrm{PaO} 2 / \mathrm{FiO} 2$ ratio $<300$, and/ or lung infiltrates $>50 \%$ of the lung field within 24 to 48 hours [24]. The definition of severe COVID-19 disease in this study was different from the WHO definition. We considered an oxygen need of 6 or more as a trigger for hospitalisation, and thus as 'severe disease', because at the time of the study there were problems with the supply of large quantities of oxygen in nursing homes and in the community. In addition, an EWS score of 7 or more during hospitalisation was regarded as an alternative definition for 'severe disease', because 7 is the threshold for prompt emergency medical assessment and may require transfer of the patient to a critical care service [16].

Finally, it is challenging to translate the continuous scores of the CFS into a cut-off score that predicts unfavourable disease outcome and that should be used as a threshold in clinical decisions regarding hospital admission or admission to ICU. This study showed that higher frailty scores predispose to worse outcomes. In our study there were no patients that died in the CFS 1-4 group. The mortality rose from $15.6 \%$ in the CFS $5-6$ group to $41.2 \%$ in the CFS 7-9 group. This underlines the chosen cut-off scores of 5 to 7 in the algorithms mentioned above [3-5], although caution is warranted. Decision algorithms should not be interpreted as a mandatory decision guides, but should serve as guidance for wellconsidered clinical decisions. When considering whether or not to upgrade the level of care, one should also take into account the patients' previous hospitalisation history, the patients' personal preferences and comfort-level. Available resources in longterm care facilities or home care should also be considered: availability of skilled personnel in adequate 
numbers, available drugs, PCR swabs, oxygen supply, protective equipment for health care workers, and possibility of isolation to prevent further spread of the disease [25].

There are several limitations to this study. First, it is a single centre study with a small study sample. This limits the power of the analysis and the generalisability of the results. Unfortunately, we only received completed online geriatric assessment questionnaires in about half of all potential candidates. Implementation of the online questionnaire during a period of crisis was challenging: contacting the family to complete the questionnaire was not always considered a priority by the responsible team. Another factor that could have played a role is that older relatives of patients may not have had access to the internet. Second, the study included patients on admission to low-care COVID-19 units. Patients who died during a primary ICU stay were not included in the study. Moreover, there may have been referral bias due to published decision algorithms with triage criteria for hospital or ICU admission. In addition, therapeutic restriction codes in patients with dementia, severe comorbidity or frailty, may have influenced therapeutic decisions and mortality rates. Third, all patients were admitted to a COVID-19 hospitalisation unit but only $90 \%$ of included patients had a positive PCR test. Some PCR-negative patients, whose diagnosis was based solely on the clinical picture and a chest CT scan, were also included. However, we excluded PCR-negative patients with alternative diagnoses during admission. An argument for including PCR-negative patients without alternative diagnoses is the fact that the sensitivity of the PCR test is only 60 to $70 \%$, so a negative test does not rule out a COVID-19 infection [26]. Fourth, clinical symptoms other than respiratory symptoms on admission, as well as laboratory findings and radiographic characteristics were not analysed in this study. Neither was the applied therapy included in the analysis. At the time of the study, the standard therapy for COVID-19 disease in our hospital (based on national guidelines) consisted of initiating hydroxychloroquine and ceftriaxone [27]. The strength of this study is the fact it considers ageing-related risk factors such as frailty and that the findings of this study can help physicians in decision making for older patients.

\section{Conclusion}

The speed of the first COVID-19 wave overwhelmed health-care workers and in the midst of the crisis it was a challenge to organise well-designed clinical studies in the geriatric population. The present study, although carried out in a small sample, suggests that the risk of death from COVID-19 disease is more related to 'ageing-related' risk factors, e.g. frailty and cognitive decline, than to 'conventional' risk factors, such as gender, obesity and specific comorbidities.

Note

1. The authors considered 6-week mortality, but all deceased patients died in-hospital.

\section{Acknowledgments}

The authors would like to thank Annouschka Laenen for providing statistical advice and the medical student trainees who helped collect the data.

\section{Disclosure statement}

The authors report no declarations of interest.

\section{Funding}

This work was not supported by a funding agency.

\section{Notes on contributor}

Katleen Fagard is a staff member at the Geriatrics department of the University Hospitals Leuven, Belgium and is currently a pursuing a PhD in Biomedical Sciences at the KU Leuven University, Department of Public Health and Primary Care. Her research focuses on perioperative care for older patients. Evelien Gielen is staff member at the department of Geriatrics and at the Centre for Metabolic Bone Diseases in UZ Leuven, Belgium, and assistant professor at the Gerontology and Geriatrics division, Department of Public Health and Primary Care, KU Leuven. Her research focuses on sarcopenia and osteoporosis in the oldest old. Mieke Deschodt is postdoctoral research fellow at the at the Gerontology and Geriatrics division, Department of Public Health and Primary Care, KU Leuven and assistant professor at the Faculty of Medicine, UHasselt in Belgium. Her research focuses on the development, implementation and evaluation of nurse-led models of care for frail older adults. Els Devriendt is Head nurse at the Geriatrics department of the University Hospitals Leuven, Belgium. She performed a Doctoral thesis on "Organisational aspects of the care of older patients in the emergency department" at the Academic Centre for Nursing and Midwifery, Department of Public Health and Primary Care, KU Leuven, Belgium and she is involved in research on models of healthcare for older persons. Johan Flamaing is head of the Geriatrics department of the University Hospitals Leuven and professor at the Gerontology and Geriatrics division, Department of Public Health and Primary Care, KU Leuven. His research focuses on infectious diseases in older patients and models and quality of healthcare for older persons.

\section{ORCID}

Katleen Fagard (D) http://orcid.org/0000-0001-5886-4432 Evelien Gielen (D) http://orcid.org/0000-0002-8985-1201 Mieke Deschodt (D) http://orcid.org/0000-0003-1560-2277 Johan Flamaing (D) http://orcid.org/0000-0003-3749-5433 


\section{Geolocation information}

Belgium.

\section{References}

[1] European Centre for Disease Prevention and Control. Event background COVID-19. 2020 [cited 2020 Sept 5]. Available from: https://www.ecdc.europa.eu/en/ novel-coronavirus/event-background-2019

[2] Yi Y, Lagniton PNP, Ye S, et al. COVID-19: what has been learned and to be learned about the novel coronavirus disease. Int J Biol Sci. 2020;16(10):1753-1766.

[3] National Institute for Health and Care Excellence (NICE). COVID-19 rapid guideline: critical care in adults. 2020 [cited 2020 Sept 5]. Available from: https://www.nice.org.uk/guidance/ng159/resources/ covid19-rapid-guideline-critical-care-in-adults-pdf $-66141848681413$

[4] Belgian Society of Intensive care medicine. Ethical principles concerning proportionality of critical care during the 2020 COVID-19 pandemic in Belgium: advice by the Belgian Society of Intensive care medicine - update 26-03-2020. [cited 2020 Sept 5]. Available from: http://www.siz.be/wp-content /uploads/COVID_19_ethical_E_rev3.pdf

[5] Belgian Society for Gerontology and Geriatrics. Beslissing opname bij mogelijk COVID positieve WZC bewoner/Arbre décisionnel admission suspicion COVID-19 résident MRS. 2020. Available from: https:// geriatrie.be/media/2020/03/2020-03-19_Beslissingopname-bij-mogelijk-COVIDpos-WZC-bewoner.pdf/ https://geriatrie.be/media/2020/03/2020-03-19_ Arbre-decisionnel-admission-suspicion-COVID-19resident-MRS_SBGG.pdf

[6] Rockwood K, Song X, MacKnight C, et al. A global clinical measure of fitness and frailty in elderly people. CMAJ. 2005 Aug 30;173(5):489-495.

[7] Muscedere J, Waters B, Varambally A, et al. The impact of frailty on intensive care unit outcomes: a systematic review and meta-analysis. Intensive Care Med. 2017 Aug;43(8):1105-1122.

[8] Zhou F, Yu T, Du R, et al. Clinical course and risk factors for mortality of adult inpatients with COVID-19 in Wuhan, China: a retrospective cohort study. Lancet. 2020 Mar 28;395(10229):1054-1062.

[9] He F, Deng Y, Li W. Coronavirus disease 2019: what we know? J Med Virol. 2020 July;92(7):719-725.

[10] Xu L, Mao Y, Chen G. Risk factors for 2019 novel coronavirus disease (COVID-19) patients progressing to critical illness: a systematic review and meta-analysis. Aging (Albany NY). 2020 June 23;12(12):12410-12421.

[11] Jordan RE, Adab P, Cheng KK. Covid-19: risk factors for severe disease and death. BMJ (Clin Res Ed). 2020 Mar 26;368:m1198.

[12] Fang $X, \mathrm{Li} \mathrm{S}, \mathrm{Yu} \mathrm{H}$, et al. Epidemiological, comorbidity factors with severity and prognosis of COVID-19: a systematic review and meta-analysis. Aging (Albany NY). 2020 July 13;12(13):12493-12503.

[13] World Health Organization. Obesity: preventing and managing the global epidemic. Report of a WHO consultation. World Health Organ Tech Rep Ser. 2000;894:i-xii,1-253.

[14] Charlson ME, Pompei P, Ales KL, et al. A new method of classifying prognostic comorbidity in longitudinal studies: development and validation. J Chronic Dis. 1987;40(5):373-383.

[15] Charlson M, Szatrowski TP, Peterson J, et al. Validation of a combined comorbidity index. J Clin Epidemiol. 1994 Nov;47(11):1245-1251.

[16] Royal College of Physicians. National early warning score (NEWS) 2: standardising the assessment of acute-illness severity in the NHS. Updated report of a working party. London: RCP; 2017 [cited 2020 Sept 5]. Available from: https://www.londonccn. nhs.uk/media/1383/news2-executive-summary_0. pdf

[17] Fried LP, Ferrucci L, Darer J, et al. Untangling the concepts of disability, frailty, and comorbidity: implications for improved targeting and care. J Gerontol A Biol Sci Med Sci. 2004 Mar;59(3):255-263.

[18] Bonanad C, García-Blas S, Tarazona-Santabalbina F, et al. The effect of age on mortality in patients with COVID-19: a meta-analysis with 611,583 subjects. J Am Med Dir Assoc. 2020 July;21(7):915-918.

[19] Cosco TD, Best J, Davis D, et al. What is the relationship between validated frailty scores and mortality for adults with COVID-19 in acute hospital care? A systematic review. Age Ageing. 2021 Jan 14. DOI:10.1093/ageing/afab008

[20] De Smet R, Mellaerts B, Vandewinckele $H$, et al. Frailty and mortality in hospitalized older adults with COVID-19: retrospective observational study. J Am Med Dir Assoc. 2020 July;21(7):928-932.e1.

[21] Hewitt J, Carter B, Vilches-Moraga A, et al. The effect of frailty on survival in patients with COVID-19 (COPE): a multicentre, European, observational cohort study. Lancet Public Health. 2020 Aug;5(8):e444-e451.

[22] Covino M, De Matteis G, Santoro M, et al. Clinical characteristics and prognostic factors in COVID-19 patients aged $\geq 80$ years. Geriatr Gerontol Int. 2020 July;20(7):704-708.

[23] Jain V, Yuan JM. Predictive symptoms and comorbidities for severe COVID-19 and intensive care unit admission: a systematic review and meta-analysis. Int J Public Health. 2020 June;65(5):533-546.

[24] Report of the WHO-China Joint Mission on Coronavirus Disease 2019 (COVID-19). [cited 2020 Sept 5]. Available from: https://www.who.int/docs/default-source/coro naviruse/who-china-joint-mission-on-covid-19-finalreport.pdf

[25] Perrotta F, Corbi G, Mazzeo G, et al. COVID-19 and the elderly: insights into pathogenesis and clinical decision-making. Aging Clin Exp Res. 2020;16:1-10.

[26] Lithander FE, Neumann S, Tenison E, et al. COVID-19 in older people: a rapid clinical review. Age Ageing. 2020 July 1;49(4):501-515.

[27] Sciensano. Interim clinical guidance for adults with suspected or confirmed COVID-19 in Belgium. 2020 [cited 2020 Sept 5]. Available from: https://covid-19. sciensano.be/sites/default/files/Covid19/COVID-19_ InterimGuidelines_Treatment_ENG.pdf 